\title{
Study on College Students Carrying Out Leisure Sports Activities and Entrepreneurship Based on the State's Vigorous Development of Sports Industry
}

\author{
Zhongjing Zhang ${ }^{1} \&$ Xiaodong $\mathrm{Chu}^{1}$ \\ ${ }^{1}$ Institute of Physical Education, Qingdao University of Science and Technology, Qingdao, China \\ Correspondence: Xiaodong Chu, Institute of Physical Education, Qingdao University of Science and Technology, \\ No.99 Songling Road, Qingdao, China. E-mail: xiaodongchu@126.com
}

Received: May 17, $2018 \quad$ Accepted: June 22, $2018 \quad$ Online Published: August 31, 2018

doi:10.5539/ass.v14n9p38

URL: https://doi.org/10.5539/ass.v14n9p38

\begin{abstract}
Under the background of the country's vigorous development of the sports industry, the sports industry ushered in unprecedented opportunities and prospects. This thesis makes a comprehensive analysis of the marketing tactics of college students' venture project- Jingyang Sihai Interesting Sports through the theory. We analyzed the problems existing in the project, the current status of the development, and clarified the goals and directions for the future development of Jingyang Sihai Interesting Sports. Although Jingyang Sihai Sports has successfully hosted many interesting projects, it also highlights many issues such as low brand value and lack of competitiveness, which are detrimental to its development. Therefore, to improve its shortcomings in the future development and carry forward its strengths, Jingyang Sihai Sports ultimately become the industry leader.
\end{abstract}

Keywords: entrepreneurship, leisure sports, opportunities, strategies

\section{Introduction}

\subsection{Research Policy Background}

At present, with the improvement of the level of residents and the rapid growth of the national economy, our country has already revealed the internal demand for the vigorous development of the sports industry. The development of the sports industry has also been developed to the national level. In recent years, the country has clarified that it is necessary to vigorously develop the sports industry, and the development of the sports industry will stimulate the enthusiasm of the people to participate in sports and promote the further improvement of the national constitution. In order to speed up the development of the sports industry, the General Office of the State Council issued "the Guidance Opinions on Accelerating the Sports Industry" (No. 22) in 2010; the State Council issued "Opinions on Accelerating the Promotion of Sports Consumption in the Sports Industry" (No. 46). It is mentioned that the total size of the sports industry exceeds five trillion in 2025 and the national fitness program has been upgraded to a national strategy. The State General Administration of Sport officially released the "13th Five-Year Plan" for the development of the sports industry to achieve the total scale of more than three trillion on July 13, 2016. The successive launch of the above documents can clearly show that the importance attached to the development of the sports industry by the state and the government has reached an unprecedented height, and a clear direction of development and development goals have been proposed (Wang, 2017).

\subsection{The Purpose of the Study}

Under the background of the country's vigorous development of the sports industry, the sports industry ushered in unprecedented opportunities and prospects. Therefore, as a contemporary college student, we must also respond to the call of the country and the government to make great efforts to develop the sports industry, accelerate the promotion of consumption, promote the national fitness, and launch an undertaking and research on the interesting sports and sports for the country (Li, 2015; Zhao \& Zhou, 2016).

Each year, both the school and the company's companies hold sporting events (Zhou \& Qi, 2007). However, due to the fixed number of participants and lack of entertainment the traditional sports games are boring. According to this situation, in order to speed up the reform and development of China's sports industry, respond to the national fitness program, and further improve the national constitution, we should vigorously carry out the 
tabloid sports. As a kind of sport with a large number of participants, high safety, strong entertainment, and high enthusiasm, tabloid sports breaks through simple and boring sports such as "running", "jumping" and "casting". The development of tabloid sports has led to a general increase in enthusiasm and an increased sense of collective honour (Tong, Meng, \& Chai, 2014). At the same time, it also creates new understanding of sports, gains happiness in sports, and is physically and spiritually satisfied (Wang, Kuang, \& He, 2016).

\subsection{Research Content of This Article}

Through the analysis and study of the theory of the marketing strategy used in my entrepreneurial project and the SWTO analysis of the tabloid sports project of Jingyang Sihai Sports in the analysis process, we point out the strengths and weaknesses of the fun projects of Jingyang Sihai Interesting Sports and the marketing used Strategy. Through the development of current interesting sports, we will formulate strategies for further development and operation.

\section{Overview of Laoshan District Jingyang Sihai Sports Culture Media Center}

\subsection{Introduction of Laoshan District Jingyang Sihai Sports Culture Media Center}

Laoshan District Jingyang Sihai Sports Culture and Media Center is engaged in the promotion and operation of sports events, planning, organization and contracting of sports events, planning of interesting sports games for corporate units, planning of school fun games, planning of outdoor activities, planning of parent-child activities, marketing planning, and leasing of event equipment. The core business is a professional sports cultural media center.

\subsection{Development status of Laoshan District Jingyang Sihai Sports Culture Media Center}

The Laoshan District Jingyang Sihai Sports Culture and Media Center was established in the first half of 2017. Through my own accumulation and strong family support and the assistance of the college teachers, I purchased a number of activities and necessary equipment for the activities. The tax bureau completed the registration and was officially launched in April. The promotion from the event to the event planning are all managed by myself. When the activity is executed, students and friends are invited to work part-time to help the smooth development of the event. At present, interest sports meetings and outdoor quality development projects have been launched for many companies and school associations. Due to the College Students' Entrepreneurship, the process also encountered a lot of problems, and currently both in activity planning and ending up, it has been more efficient than before by the accumulation of experience.

\subsection{Introduction to the Fun Sports Project of Jingyang Sihai Sports Culture Media Center in Laoshan District}

As a high participatory activity, the interest movement can strengthen the exchange and cooperation among the participants and enhance the friendship between the participants. The past competitive sports should be paid attention to the individual to the collective, the unity and cooperation of the students and the cohesion of the enterprise. This is especially important for schools and enterprises.

Interest sports are no longer the "manual labor" in the past, which greatly improves people's enthusiasm for participation. For the school, improve the physical quality of the students, set up the concept of lifelong exercise; for the enterprise, the fun movement will relieve the employees in the busy work, release the pressure to improve the efficiency of work.

The characteristic of interest sports is the participation of all people. Men, women, old and young are all suitable to win by chance. Therefore, the development of the fun games has created a stage for the masses of people to participate in sports, physical fitness and enjoyment. This is in line with China's national fitness strategy, promoting the reform and development of China's sports industry, and accelerating sports industry to promote consumption.

\section{SWOT Analysis of Marketing Environment of Interesting Sports Events in Jingyang Sihai Sports Culture and Media Center}

\subsection{Introduction of SWOT Analysis Method}

The SWOT analysis method is an enterprise strategic analysis method which is based on the established internal conditions of the enterprise to find out the advantages, disadvantages and core competitiveness of the enterprise. The strategic internal factors: S represents strength, W represents weakness, and external factors: O represents opportunity, T represents threat.

Strength is the internal factors of the organization, including: favorable competitive situation, sufficient financial sources, good corporate image, technical force, scale economy, product quality, market share, cost advantage, 
advertising offensive and so on.

Weakness refers to the relative weakness in competition. It is also the internal factors of the organization, including: equipment aging, management confusion, lack of key technology, backward research and development, shortage of funds, poor management, product backlog, poor competitiveness and so on.

Opportunity is the external factor of the organization, including new products, new market, new demand, market barrier release, competitor error and so on.

Threat is also an external factor in the organization, including: new competitors, the increase in replacement products, market tightening, industry policy changes, economic recession, customer preference changes, emergencies and so on.

3.2 SWOT Analysis of Marketing Environment of Interesting Sports Events in Jingyang Sihai Sports Culture and Media Center

\subsubsection{Advantages Analysis}

The unique market positioning, low cost and high stability, innovative service content, attractive, participatory and interesting are the great advantages of the project. It is embodied in the unique market positioning characterized by entertainment experience and interesting sports.

There are many cost advantages. The project has the characteristics of low cost and stable product. The main cost is artificial cost and the product is low in the personal skill requirements for the activity executors. After short-term training, it can carry out the activities smoothly, which reduces the cost of the product more. Meanwhile, there are also many product advantages. The project innovation to make participants feel fresh through a variety of special new equipment. The sports include many projects and are simple, interesting and stimulating. In addition, sports participation is strong and controllable under the premise of security.

\subsubsection{Disadvantages Analysis}

The first is propaganda inferiority. At present, the propaganda way of Jingyang Sihai sports interesting project is relatively single by the WeChat public number push and the recommendation of teachers and friends to publicize so that the service project can't be quickly publicize.

The second is the disadvantage of capital. The company is in the entrepreneurial stage. The shortage of funds has made it impossible to purchase more equipment and equipment to enrich the activities. This has resulted in a single service product and lack of innovation and the equipment cannot be replaced in time after aging and breakage.

The last is the management disadvantage. At present, I am the only person in the company. All the work is done by me alone. Sometimes there is a lot of work and the time cannot guarantee high efficiency.

\subsubsection{Opportunity Analysis}

At present, China's sports industry is developing at a rapid rate. To speed up the development of the sports industry, the General Office of the State Council issued the "Guidance on Accelerating the Sports Industry" (No.22) in 2010; and the State Council issued "Accelerating Sports Consumption for Sports Consumption in 2014. Several opinions (No. 46) mentioned that the total size of the sports industry exceeds five trillion in 2025 and that national fitness will be promoted as a national strategy. On July 13, 2016, the State General Administration of Sport officially released the "13th Five-Year Plan for the Development of Sports Industry". The planning aims to achieve a total scale of more than three trillion sports industry. We will vigorously attract social investment and encourage social capital to enter the sports industry, build sports facilities, develop sports products, and provide sports services. It is in line with the new situation in which the country is now vigorously developing the sports industry; there are professional teachers who provide guidance and master some social resources to provide greater help for project implementation.

\subsubsection{Threat Analysis}

Since the Jingyang Sihai interesting sports project is carried out, the social influence is small and the publicity is not enough, so many customers who have interesting activities have not chosen us. At present, there are strong competitors in the market, and the reproducible products of this kind of products are strong. It is necessary to optimize the products and determine their own core competitiveness. 


\section{Problems Existing in Jingyang Sihai Interesting Sports Project}

\subsection{Shortage of Products}

Jingyang Sihai sports is in the initial stage of entrepreneurship. Due to the limited purchase of equipment and equipment, the games that customers can choose are limited. Compared with the competitors, the products are single, unable to meet some customers' requirements, and the market competitiveness is not enough. We cannot update and enrich our products in time. Now we have cooperated with Bird Technology Co., Ltd. and Haicheng Bangda Logistics International Co., Ltd. However, if the cooperation product is a previous project, the chances of the customer's choice will be greatly reduced.

\subsection{Limited Coverage of Promotional Channels}

Jingyang Sihai interesting sports project is mainly publicizing by WeChat public number, micro-blog, teachers, classmates and friends. Because it has no cooperation with the media, we cannot carry out a variety of publicity, and the propaganda speed is slow. There is a certain degree of restriction, therefore the market influence is not enough.

\subsection{Price Positioning}

The charge standard for Jingyang Sihai interesting project is based on the market price. Although there are more advantages in prices than competitors, the project are still relatively expensive compared to some companies with a reasonable profit. The demand customers are mainly concentrated in high-profit corporate units and schools.

\section{Current Development of Interesting Sports}

\subsection{Interesting Sports on Campus}

There are numerous students and so it is difficult to organize activities. Due to the large population base, there are numerous middle and elementary school students. However, there are a limited number of physical education teachers in school. School teachers subconsciously still use traditional track and field games as their main activity. The organizing experience of interesting activities is relatively lacking. The development mode and development ideas of the fun sports games are not clear enough, and detailed organization plans cannot be planned. In addition, the equipment in school are limited and is mainly related to physical education and traditional sports games. There is no device for performing fun activities, which makes it impossible for the fun activities to be carried out and hit the enthusiasm of the organization department to a certain extent. School leaders pay little attention to the educational value of fun games, which caused the development of interesting activities to be smashed into the cradle. Affected by traditional games, it is believed that the games are for students to play, they do not realize the value of education, and there is no deeper improvement in the overall quality of students. The school has also carried out interesting activities in the past, but the lack of innovation in sports projects is not novel enough to result in low student motivation.

\subsection{Interesting Sports in Enterprises}

Many leaders of enterprises or organizations pay little attention to the cultural life of employees, and don't realize that active activities of employees have a positive effect on cohesion and work efficiency. As a result, the fun activities of enterprises or organizations cannot be carried out. Activities budget expenditure is too small to carry out fun activities, so most of the enterprises that hold interesting activities are mainly high-income units. The enterprises lack the relevant equipment for organizing fun games, which makes it impossible for the fun activities to be carried out and combat the enthusiasm of the organization department to some extent. The company's organization department has limited personnel and lack of experience, unable to plan a detailed organization program. Some units have also carried out interesting activities in the past, but the lack of innovation in sports projects is not novel enough to enable employees to truly feel the joy of fun sports.

\section{The Future Development Strategy of Jingyang Sihai Interesting Sports Project}

\subsection{Shortage of Product}

In order to solve the problem of shortage of products, we should maintain the diversification of products, innovate and enhance the competitiveness of products, attract investment, give funds to support rich products, cooperate with peers, lease equipment, and aim to maintain the diversity of products. We should constantly improve the added value of interesting sports and improve the service level, so as to ensure the priority preference of customers in carrying out interesting sports activities. 


\subsection{Publicity Channel Problem}

After a certain amount of initial stage of Jingyang Sihai Interesting Sports project, it will intensify propaganda, cooperate with television stations, publishing houses and other media to expand publicity channels and increase market influence.

\subsection{Pricing Strategy}

Since the Jingyang Sihai Interesting Sports project is a start-up project, the current cost is calculated based on the number of people. Therefore, a lower price is used to quickly attract a large number of customers and increase the market share. However, this has caused a large amount of capital investment in the previous period, which is not conducive to the long-term development of the Jingyang Sihai Interesting Sports project. Hence, when the project is mature, the pricing strategy will use its psychological pricing strategy, adopting the mantissa pricing method and combining sales promotion strategies to carry out supplementary pricing

\subsection{Development Goals}

Now the Jingyang Sihai Interesting Sports project should be more concerned about how to improve the product's core competitiveness and high added-value, develop more extended products, improve its service industry chain, and maintain the stable growth. Focus on customer needs and explore potential needs to dominate the market. To ensure its own advantages, reduce the duplication of products, to achieve the authority and professionalism of the industry, so that customers have priority in future selection of such products.

\section{Acknowledgments}

Project supported by the College Students Innovation and Entrepreneurship Training Program of Qingdao University of Science and Technology

\section{References}

Li, M. X. (2015). Research on reform and innovation of track and field games and fun games in primary and secondary schools. Manager Journal, 4, 385.

Tong, W. X., Meng, Y. D., \& Chai, X. F. (2014). Feasibility study of developing fun games in high-tech middle schools in Zhengzhou city. Contemporary Sports Technology, 4(28), 84-85.

Wang, D., Kuang, J., \& He, L. H. (2016). Research on the current situation and significance of the fun games of primary and secondary school students and relevant countermeasures -- take the example of some middle schools and primary schools in Yongchuan District of Chongqing City. Contemporary Sports Technology, 6(31), 112-113.

Wang, K. Y. (2017). The research on the necessity of developing sports industry in China in the new period. Contemporary Sports Technology, 7(10), 235-237.

Zhao, Z. Y., \& Zhou, L. (2016). Analysis of the current situation of the fun games in Wuhan enterprises and institutions. Contemporary Sports Technology, 6(14), 88-89.

Zhou, M. X., \& Qi, S. C. (2007). Sports consumption and national fitness. Market Modernization, 35, 135-136.

\section{Copyrights}

Copyright for this article is retained by the author(s), with first publication rights granted to the journal.

This is an open-access article distributed under the terms and conditions of the Creative Commons Attribution license (http://creativecommons.org/licenses/by/4.0/). 